\title{
Calculating voltage magnitudes and voltage phase angles of real electrical networks using artificial intelligence techniques
}

\author{
Meriem Fikri, Omar Sabri, Bouchra Cheddadi \\ Laboratory RITM EST, University Hassan II, Morocco
}

\begin{tabular}{l}
\hline \hline Article Info \\
\hline Article history: \\
Received Mar 5, 2020 \\
Revised May 11, 2020 \\
Accepted May 24, 2020 \\
\hline
\end{tabular}

\section{Keywords:}

Adaptive neuro-fuzzy inference Artificial intelligent methods Artificial neural network Electrical network Power flow analysis

\begin{abstract}
In the field of electrical network, it is necessary, under different conditions, to learn about the behavior of the system. Power Flow Analysis is the tool per excellent that allow as to make a deep study and define all quantities of each bus of the system. To determine power flow analysis there is a lot of methods, we have either numerical or intelligent techniques. Lately, researchers always work on finding intelligent methods that allow them to solve their complex problems. The goal of this article is to compare two intelligent methods that are capable of predicting quantities; artificial neural network and adaptive neuro-fuzzy inference system using real electrical networks. To do that we used few significant discrepancies. These methods are characterized by giving results in real time. To make this comparison successful, we implemented these two methods, to predict the voltage magnitudes and the voltage phase angles, on two Moroccan electrical networks. The results of the comparison show that the method of adaptive neuro-fuzzy inference system have more advantages than the method of artificial neural network.
\end{abstract}

Copyright $(2020$ Institute of Advanced Engineering and Science. All rights reserved.

\section{Corresponding Author:}

Meriem Fikri,

Laboratory RITM EST,

Hassan II University,

Route d'Eljadida, KM 7, N1, Casablanca, Morocco.

Email: meriem.fikri@gmail.com

\section{INTRODUCTION}

The complexity and the growth in size of electrical systems have necessitated the use of reliable methods which can solve the problem of power flow analysis. The important development in hardware and software technologies have transformed the solution of power flow analysis from complex task to a simple process capable of getting very good solution. The techniques of artificial intelligence have made their own way into a lot of fields in our lives.

Artificial intelligent (AI) is the ability of machines to use algorithms to learn from data, and use what has been learned to make decisions like a human would. Unlike humans, though, AI powered machines don't need to take breaks or rest and they can analyze massive volumes of information all at once. The ratio of errors is also significantly lower for machines that perform the same tasks as their human counterparts. Another benefit of $\mathrm{AI}$ is that it allows machines and robots to perform tasks that humans consider to be difficult, boring, or dangerous. In turn, this will enable humankind to do things that were once thought impossible [1].

A neural network (NN) is a computing systems inspired by the biological neural networks. Neural networks have a lot of types, some of them are simple and some others are complex [2]. artificial neural networks (ANN) are effective in dealing with tricky issues, unclear problems or highly nonlinear with a lot of variables [3]. Adaptive neural fuzzy inference system (ANFIS) uses the learning 
algorithm of the artificial neural network and it is based on fuzzy logic modeling. Since it combines between the properties of both, it can benefits from the advantages of each method [4].

The purpose of estimating the state of an electrical system is to process the available information and produce the best possible estimate of the actual state of this system. It is a numerical processing scheme that provides a real-time database for many specific functions. With the state estimator we can predict the values of voltage magnitudes and voltage phase angles and get similar results as the real one [5]. In this paper we aim to predict the voltage magnitudes and the voltage phase angles at each bus of the two Moroccan electrical networks, in real time and with great precision using two artificial intelligent techniques and based on the analyzes we obtained from the study that we did, the method of ANFIS was the best compared to the method of the ANN in terms of calculation time and precision.

The study proposed in this paper is to predict voltage magnitudes and voltage phase angles using ANN and ANFIS and make a comparison between this two intelligent methods. A lot of articles dealt with subjects related to artificial intelligent methods in solving different problems of electrical power. In reference [6], the paper discussed the use of ANN in solving load flow problem. Other paper of reference [7], treated the study of optimal power flow using ANN. In [8], the authors used the ANFIS for power flow analysis. In [9], we have a comparison between artificial neural network and hybrid intelligent genetic algorithm in predicting the severity of fixed object crashes among elderly drivers. Reference [10] proposed a machine learning for intelligent optical networks. In [11], they applied artificial intelligence in electrical automation control. In [12], the technique of ANFIS was used for fault classification in the transmission lines. To improve a large-scale power system stability the authors make a coordination of ANFIS and type-2 fuzzy logic system-power system stabilizer [13]. ANFIS and other method were used to calculate the short-term demand forecasting of a multi-carrier energy system and to optimize its energy flow [14]. There is other methods that were used in power system, for example a modified models of PSO algorithm was used to get the estimation solution of power system state [15], in reference [16], the authors used the continuous Newton's for power flow analysis, reference [17] proposed the firefly algorithm for solving load flow problem and authors of paper [18] discussed the optimal power flow based congestion management using enhanced genetic algorithms. Reference [19] proposed a study about strengths, solutions and limitations of ANFIS.

Regarding the previous contributions, we managed to make at first an international communication and a journal article. In the communication, we discussed the use of the two numerical methods; Gauss-Seidel and Newton-Raphson and the artificial intelligence method neural networks to solve power flow problem [20]. Concerning the journal article, it dealt with three numerical methods; Gauss-Seidel, Newton-Raphson and fast decoupled in addition to the method of neural networks for analyzing the power flow [21]. We succeed to do also two other international communications. One was about predicting Moroccan real network's power flow employing the ANN [22] and the other one was about predicting the voltage magnitudes and voltage phase angles using ANFIS [23]. The main goal of this article is to compare the two intelligent methods Artificial Neural Network and Adaptive Neuro-Fuzzy Inference System using two real electrical networks.

In this article, the sections will be as follow; the first section will concentrate on bibliographic studies, where we will give an overview on power flow analysis, artificial neural network, adaptive neuro-fuzzy inference system and significant discrepancies. The second section will present the application of the two intelligent methods on the transmission systems. The last section will be dedicated for communication and discussion of the results obtained.

\section{BIBLIOGRAPHIC STUDIES}

\subsection{Power flow study}

Power system engineering is very important in electrical networks, and the calculation of power system problems presents the key of power flow analysis. The importance of the latter cannot be overstated. It is essential for predicting the behavior of power systems in the steady state and as part of the process for transient conditions. It is a fundamental tool for practicing power system engineers engaged in system planning, operation, and control [24].

The power flow problem was first solved using the simplest techniques, soon to be replaced by more sophisticated methods. The development of computer methods for power flow analysis actually benefited from the availability of only slow-speed, limited-memory computers. It was necessary always to seek techniques that would optimize their performance, such as Artificial Intelligent techniques. As a result today's commercial power flow programs offer the advantage of high-speed operation, along with the availability of cheap, high-speed computers [24]. 


\subsection{Artificial neural networks}

Artificial neural networks are computing systems inspired by observations of the biological systems. An artificial neural network can be described as mapping an input space to an output space [25]. Neurons are connected to other neurons. The Figure 1 shows a simple model of neural network, we have three neurons feed the single neuron, with one output come from the single neuron [25].

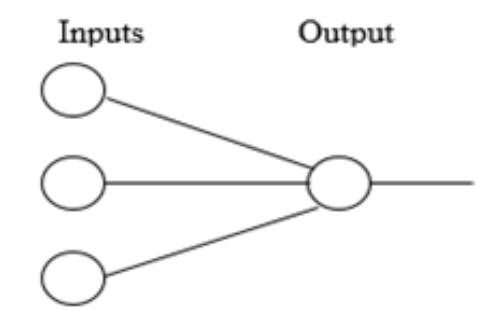

Figure 1. Model of artificial neuron

\subsubsection{Artificial neural network structure}

The basic structure of an ANN consists of artificial neurons that are grouped into layers. The most common ANN structure consists of an input layer, one or more hidden layers and an output layer. For more details check references [20-22]. There are two training methods; supervised and unsupervised. The Supervised training employs a "teacher" to help in training the network by giving it the desired output of the specific input. The most important thing is that supervised learning demands an input and a corresponding target. Unlike the supervised training, the unsupervised training doesn't use a teacher in the training process, so it doesn't need a desired output. The learning process is a somewhat open loop, with a set of adaptation rules that govern general behavior. Note that unlike the supervised-training method, the unsupervised method does not need a desired output for each input-feature vector [25].

\subsubsection{The feed forward neural network}

Backpropagation is an algorithm used in training the network for supervised learning, it is a gradient-descent approach that it uses the minimization of first-order derivatives to find an optimal solution. As the most training algorithms, the goal of backpropagation is to adjust the weights in the network to produce the desired output by minimizing output error. The training algorithm iteratively tries to force the generated outputs to the desired target output [25]. Most artificial neural network uses supervised learning as a training method, usually the backpropagation algorithm and the most common model is a feedforward neural network containing the transfer function "sigmoid" [25].

\subsection{Adaptive neuro-fuzzy inference system}

Adaptive neuro-fuzzy inference systems (ANFIS) are an integration of neural networks and fuzzy systems that can give a good modeling approach of difficult problems. ANFIS is a model that combine between the interpretability of a fuzzy inference system with the adaptability of a neural network.

\subsubsection{Adaptive neuro-fuzzy inference systems structure}

ANFIS consists of five layers, where each layer corresponds to the realization of a step of a fuzzy inference system of the Takagi Sugeno type. To get the desired performance of the adaptive system, it's important to properly select the type, the number and the parameters of the membership functions. The good choice of the ANFIS structure facilitate learning and adaptation [4]. For more details check reference [23].

\subsubsection{ANFIS's learning process}

ANFIS applies the mechanism of learning neural networks on fuzzy inference techniques. ANFIS uses a two-pass learning cycle: forward propagation and back propagation [23]. During the training process, membership functions parameters are adjusted using the backpropagation learning algorithm, or in combination with another type of algorithms such as the least square to identify the best prediction value. This combination makes it possible to build a hybrid method that ameliorates the disadvantages of each algorithm [4]. 


\subsection{Significant discrepancy}

Below, we note «yi» a value taken by an explained variable and «yi'» the value as it would have been predicted by the model. " $n$ " is the number of observations.

\subsubsection{Mean square error (MSE)}

It is the arithmetic mean of the squares of the differences between model predictions and observations. The mean squared error gives an interpretation on the relation between the regression line and the set of points. It squares the distances from the points to the regression line (these distances are the "errors").

$$
M S E=\frac{1}{n} \sum_{i=1}^{n}\left|y_{i}^{\prime}-y_{i}\right|^{2}=\frac{1}{n} \sum_{i=1}^{n} e_{i}^{2}
$$

\subsubsection{Root mean square error (RMSE)}

Root mean square error (RMSE) is the standard deviation of the prediction errors. It tells you how concentrated the data is around the line of best fit [26].

$$
R M S E=\sqrt{\frac{1}{n} \sum_{i=1}^{n} e_{i}^{2}}
$$

\section{EXECUTION OF THE METHODS}

In this article we will discuss the results obtained by using the ANN and ANFIS methods to predict voltage magnitudes (VM) and voltage phase angles (VPA) in two different electrical networks. The first network is the ONE 14 bus system and the second one is the ONE 24 bus system. These are two real electrical networks in Morocco.

\subsection{ONE 14 bus system power flow analysis}

Tests are carried out on the ONE 14 bus system; it represents a portion of the Casablanca electrical network. The Figure 2 represent this system which is composed of 3 generator buses and 11 load buses.

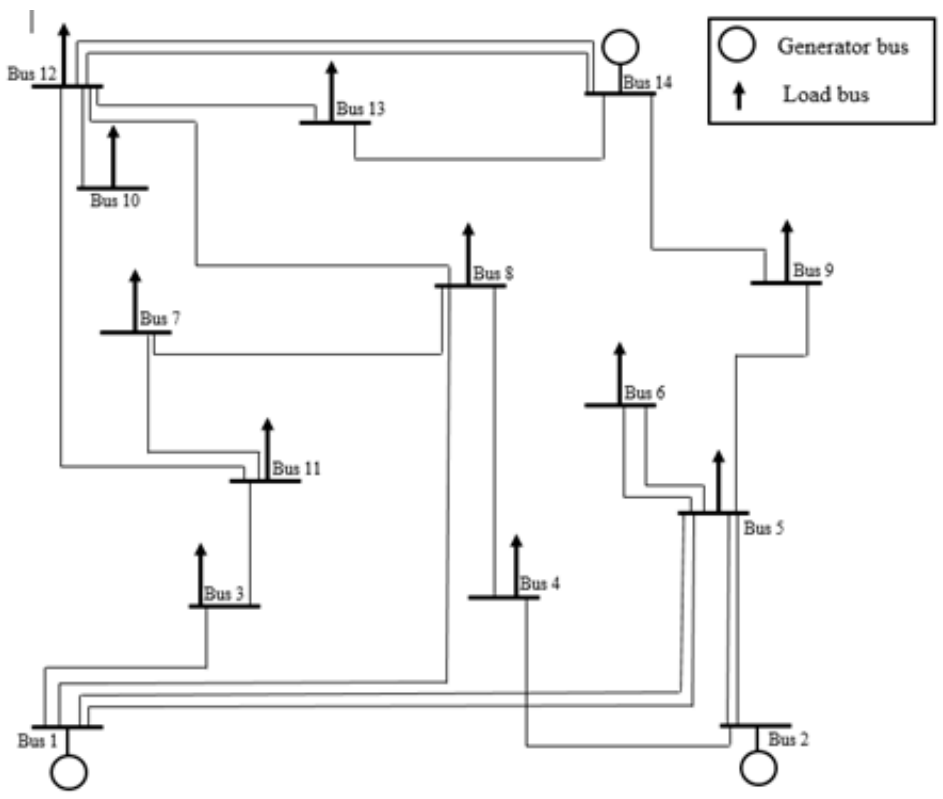

Figure 2. One line diagram of ONE 14 bus system

\subsubsection{Application of ANN}

Training phase of ANN: The Figure 3 presents the models of ANN to predict VM and VPA. At the left, we have the NN model to predict VM and at the right, the model to predict VPA. In the training phase we used the mean square error to test the performance of the ANN models. The following graphs of 
Figure 4 present that performance. The curves of train, validation and test does not show any major problem with the training. The validation and test curves are very similar. The results obtained tell that there is an excellent linear relationship between outputs and targets.
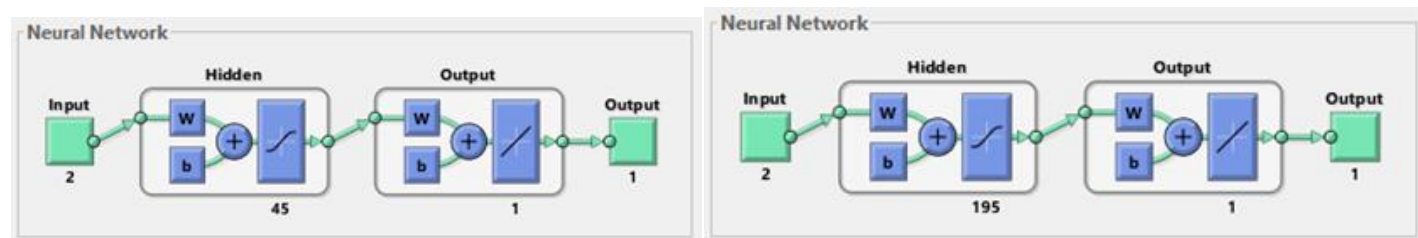

Figure 3. ANN models to predict voltage magnitudes and voltage phase angles
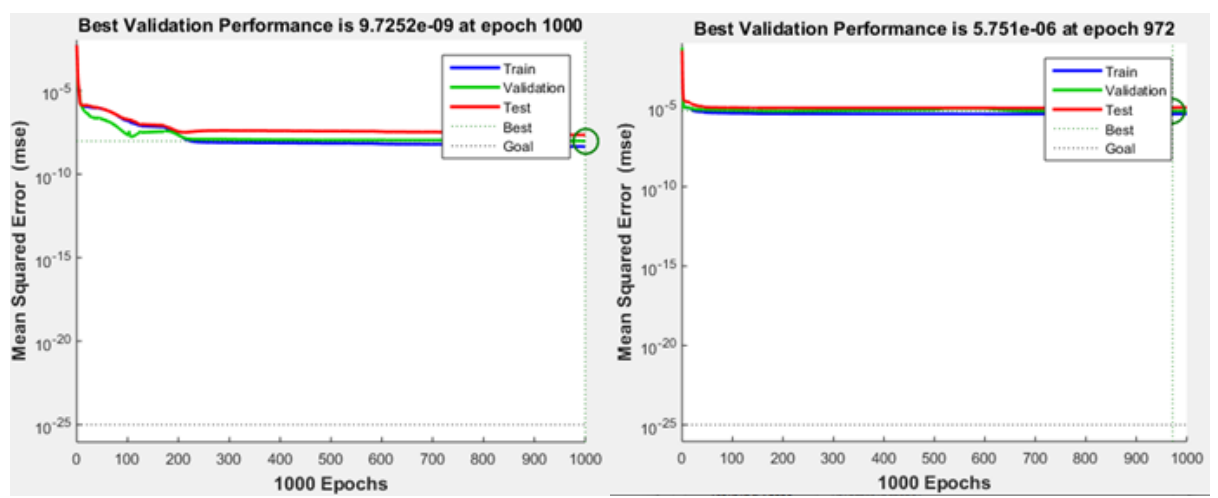

Figure 4. Training performance of the ANN models

\subsubsection{Application of ANFIS}

Training phase of ANFIS: The Figure 5 presents the models of ANFIS to predict VM and VPA. For the model at the left to predict VM, we have two inputs which are active and reactive power of load buses, we used five membership functions type "gbellmf" and one output which is VM. For the model at the right to predict VPA, we have two inputs which are active and reactive power of load buses and active power and voltage magnitudes of generator buses, we used seven membership functions type "gbellmf" and one output which is VPA. The number of epoch that we needed to get the results for VM is 95 and for VPA is 18.

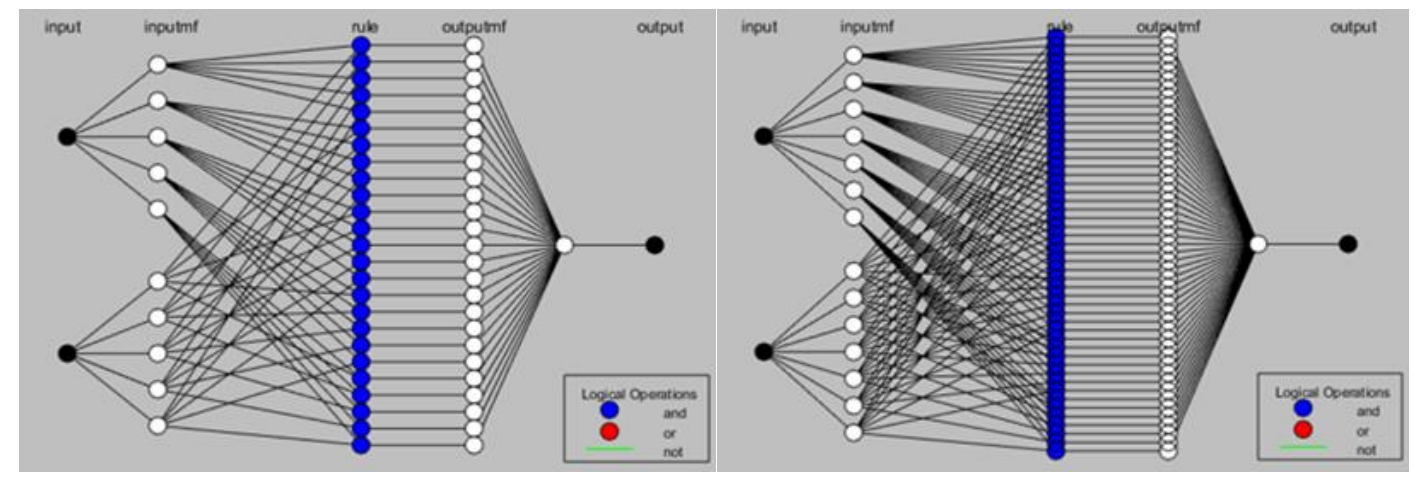

Figure 5. ANFIS models to predict voltage magnitudes and voltage phase angles

\subsubsection{Graphic Representations of voltage magnitudes and voltage phase angles}

The following graphs of Figure 6 present the VM and VPA calculated by using ANN and ANFIS methods compared to Newton Raphson method that we took as reference. 

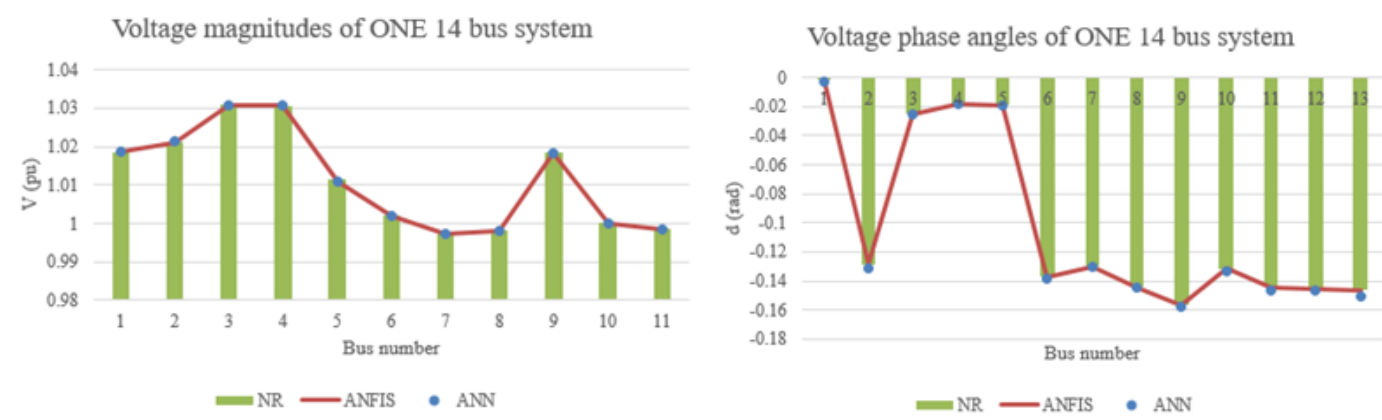

Figure 6. Graphic representations of voltage magnitudes and voltage phase angles

\subsection{ONE 24 bus system power flow analysis}

Tests are carried out on the ONE 24 bus network. The $225 \mathrm{kV}$ transmission and sub-transmission system network is composed of 24 buses with 6 generator buses and 18 load buses. The Figure 7 presents the one line diagram of this system, it is the same system used in the paper of reference [22].

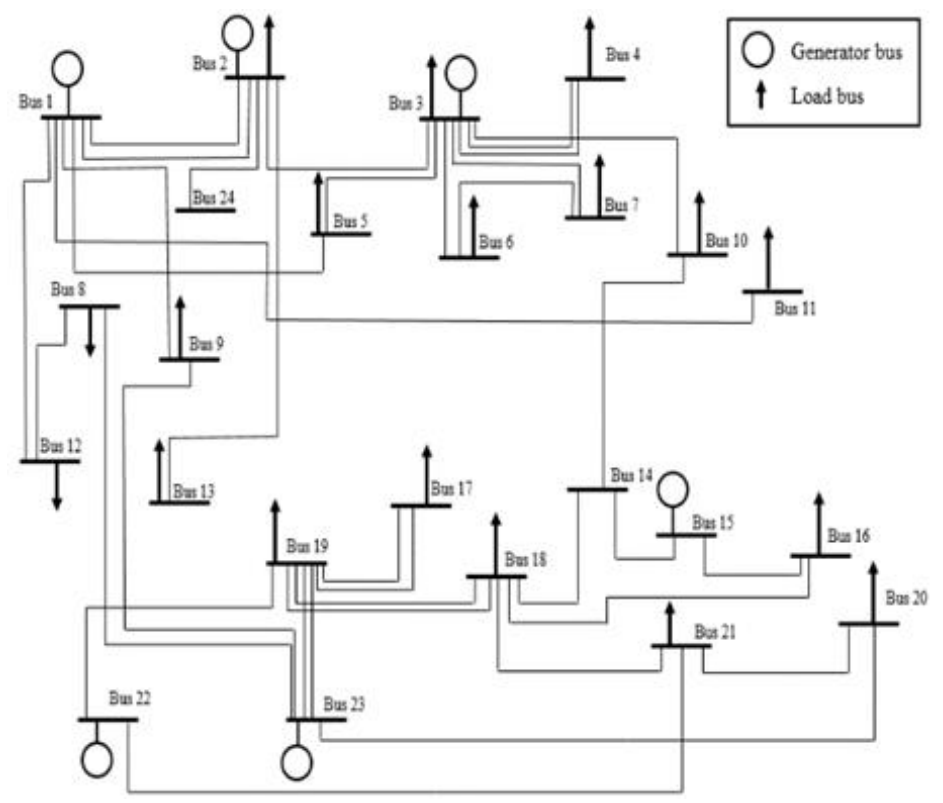

Figure 7. One line diagram of ONE 24 bus system [22]

\subsubsection{Application of ANN}

Training phase of ANN: The Figure 8 presents the models of ANN to predict VM and VPA. At the left, we have the NN model to predict VM and at the right the model to predict VPA. The curves of Figure 9 don't show any major problem with the training. The validation and test curves are very similar. The results obtained tell that there is an excellent linear relationship between outputs and targets.

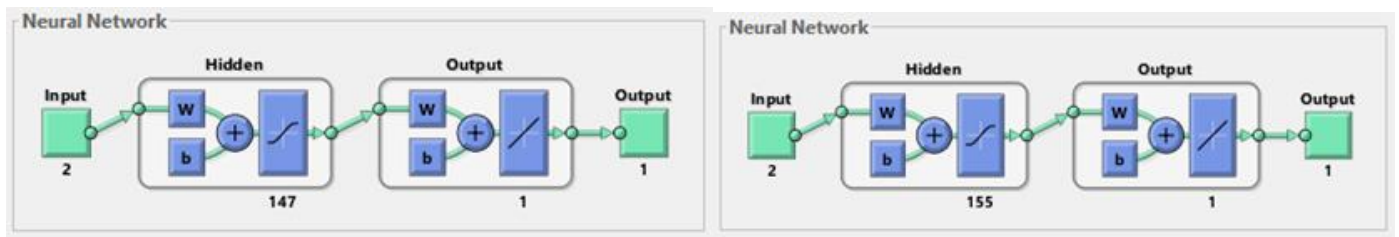

Figure 8. ANN models to predict voltage magnitudes and voltage phase angles 

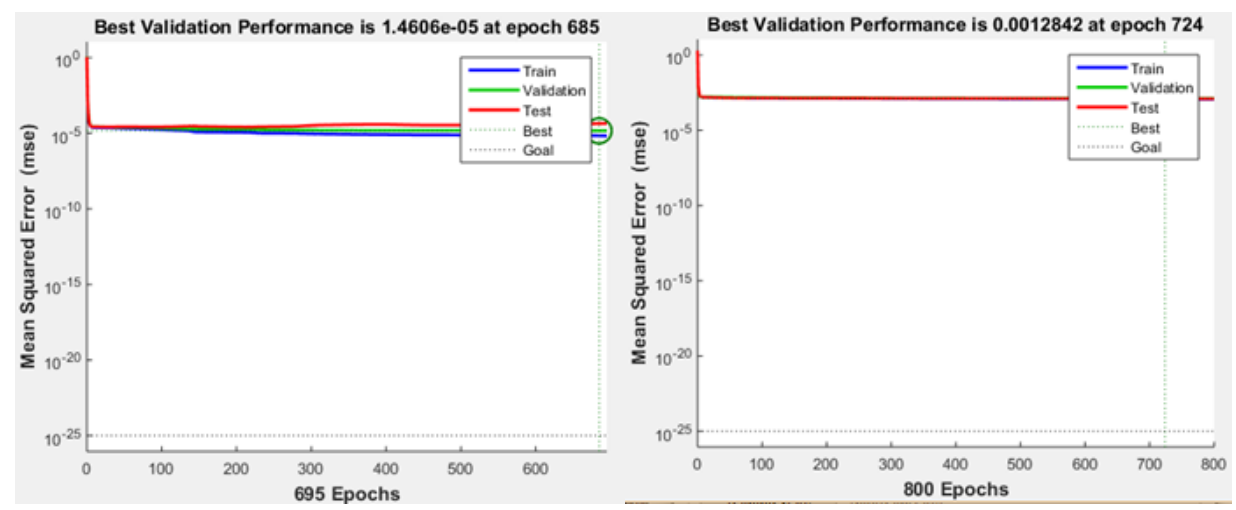

Figure 9. Training performance of the ANN models

\subsubsection{Application of ANFIS}

Training phase of ANFIS: The Figure 10 presents the models of ANFIS to predict VM and VPA. For the model at the left to predict VM, we have two inputs which are active and reactive power of load buses, we used seven membership functions type "gbellmf" and one output which is VM. For the model at the right to predict VPA, we have two inputs which are active and reactive power of load buses and active power and voltage magnitude of generator buses, we used seven membership functions type "gbellmf" and one output which is VPA. The number of epoch that we needed to get the results for VM is 80 and for VPA is 70 .

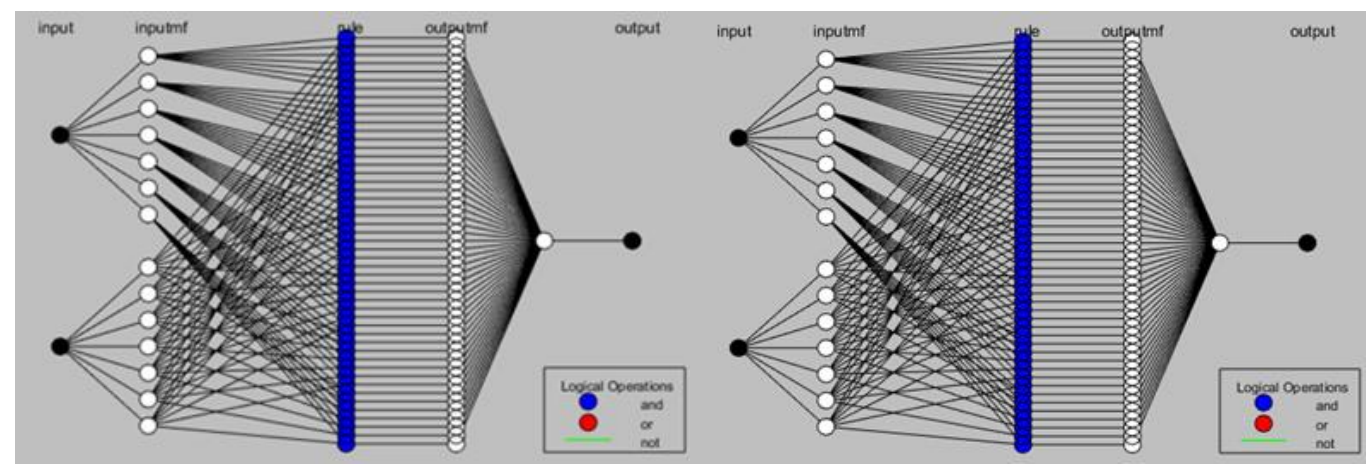

Figure 10. ANFIS models to predict voltage magnitudes and voltage phase angles

\subsubsection{Graphic representations of voltage magnitudes and voltage phase angles}

The following graphs of Figure 11 present the VM and VPA calculated by using ANN and ANFIS methods compared to Newton Raphson method that we took as reference.
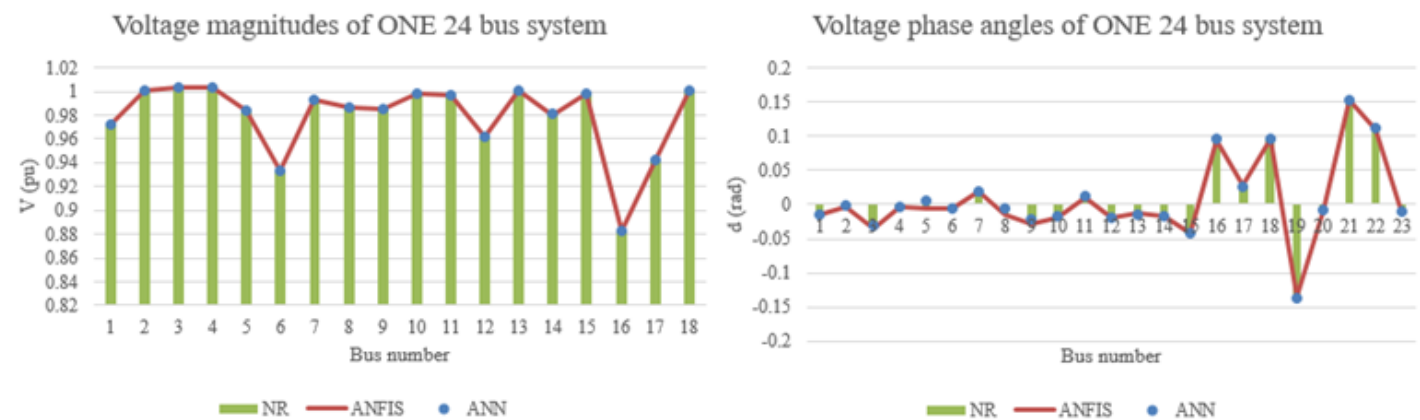

Figure 11. Graphic representations of voltage magnitudes and voltage phase angles 


\section{RESULTS INTERPRETATION}

In this section, we will compare the results that we get by implementing the two artificial intelligence methods ANN and ANFIS to predict VM and VPA.

\subsection{The training and calculation time}

The Figure 12 presents the training and calculation time. According to the graph at the left, we can clearly see that the time required by the ANN method for the model to be trained is much bigger than that required by the ANFIS method. As present the graph at the right, the calculation time required to predict the VM and the VPA by the ANFIS method is significantly smaller than the calculation time required by the ANN method.
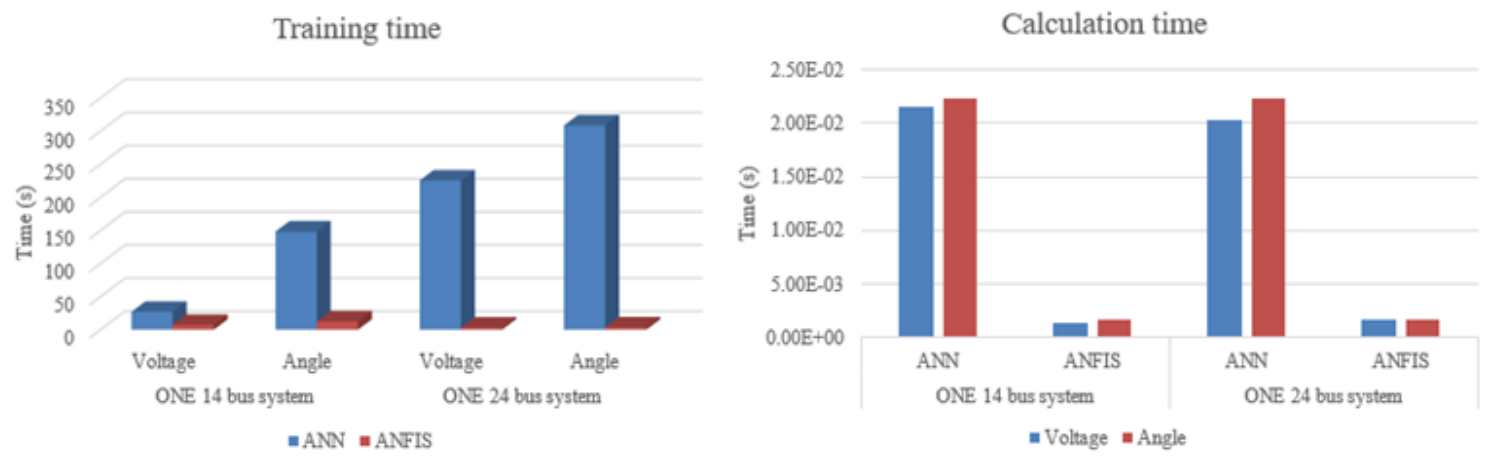

Figure 12. Graphic representations of training and calculation time

\subsection{Significant discrepancy analysis}

The Figure 13 presents the mean square error (MSE) and the root mean square error (RMSE). The smaller the means squared error, the closer we are to the real value. The bottom line is that, for our case study, it is clear that the ANFIS method is more advantageous than that of the ANN and combining between several methods has borne fruit.
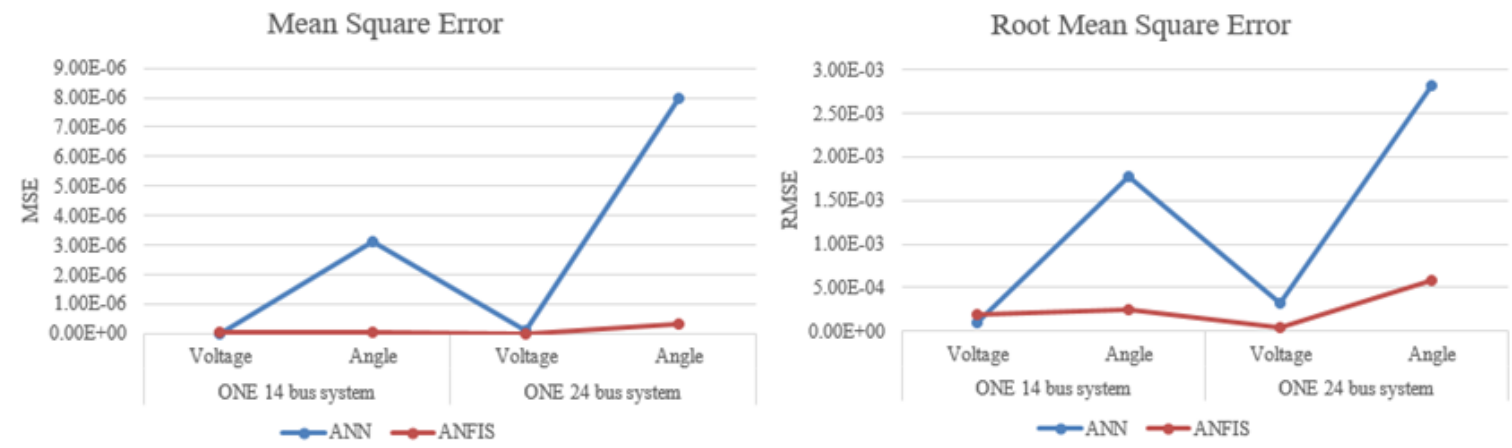

Figure 13. Graphic representations of MSE and RMSE

\section{CONCLUSION}

Electrical networks have experienced a huge growth following the development of computer and soft computing. The calculation of the power flow is necessary to define the state of the electrical network in terms of stability, reliability and economic cost. The implementation of power flow study allow us to determine all values wanted. Due to the important of this subject researchers work a lot on new methods to solve problem of power flow analysis. Using unfamiliar techniques to solve difficult problems in special field is one of the biggest challenge for researchers around the world. Implementing intelligent methods such as ANN and ANFIS to predict voltage magnitudes and voltage phase angles was not an easy task due to a lot of challenges such as the lack of historical data and the difficulties of constructing ANN and ANFIS models. 
The intelligent methods perform all the calculations to find the desired values. In this article we tried to compare between two intelligent methods ANN and ANFIS. The study shows that ANFIS is stronger compared to ANN in terms of training time, execution time and average error, which means that combining several methods is very useful to get better results in terms of time and precision. It's difficult to choose between them or to say one method is better than the other, but it depends on the nature of the problem itself and which method is perfect for it. However, intelligent methods seem useful and effective for these complex problems. Combining several intelligent methods is useful to overcome the disadvantages and amplify the advantages of each.

\section{REFERENCES}

[1] L. Rouhiainen, "Artificial intelligence 101 things you must know today about our future," Kindle Edition, 2018.

[2] V. B. Rao, "C++ Neural Networks and Fuzzy Logic," MTBooks, IDG Books Worldwide, Inc., 1995.

[3] D. Graupe, "Principles of Artificial Neural Networks," Advanced Series in Circuits and Systems, vol. 7, 2013.

[4] M. F. Azeem, "Fuzzy Inference System-Theory and Applications," Open access peer-reviewed Edited Volume, 2012.

[5] A. Gulati, et al., "Overview of State Estimation Technique for Power System Control," IOSR Journal of Electrical and Electronics Engineering (IOSR-JEEE), vol. 8, no. 5, pp. 51-55, 2013.

[6] W. A. Alsulami and R. S. Kumar, "Artificial Neural Network based Load Flow Solution of Saudi National Grid," 2017 Saudi Arabia Smart Grid (SASG), Jeddah, Saudi Arabia, pp. 1-7, 2017.

[7] L. Imen, et al., "Optimal power flow study using conventional and neural networks methods," 2015 International Conference on Renewable Energy Research and Applications (ICRERA), Palermo, Italy, pp. 1422-1427, 2015.

[8] D. Abdellah and L. Djamel, "Power Flow Analysis using Adaptive Neuro-Fuzzy Inference System," $20153 \mathrm{rd}$ International Renewable and Sustainable Energy Conference (IRSEC), Marrakech, Morocco, pp. 1-5, 2015.

[9] A. M. Amiri, et al., "A comparison between Artificial Neural Network and Hybrid Intelligent Genetic Algorithm in predicting the severity of fixed object crashes among elderly drivers," Accident Analysis and Prevention, vol. 138, pp. $105468,2020$.

[10] R. Gu, et al., "Machine learning for intelligent optical networks: A comprehensive survey," Journal of Network and Computer Applications, vol. 157, pp. 102576, 2020.

[11] L. B. Yang, "Application of Artificial Intelligence in Electrical Automation Control," Procedia Computer Science, vol. 166, pp. 292-295, 2020.

[12] T.S. Kamel and M.A.M. Hassan, "Adaptive Neuro Fuzzy Inference System (ANFIS) For Fault Classification in the Transmission Lines," The Online Journal on Electronics and Electrical Engineering, vol. 2, no. 1, pp. 164-169, 2009.

[13] A. B. Muljono, et al., "Coordination of Adaptive Neuro Fuzzy Inference System (ANFIS) and Type-2 Fuzzy Logic System-Power System Stabilizer (T2FLS-PSS) to Improve a Large-scale Power System Stability," International Journal of Electrical and Computer Engineering (IJECE), vol. 8, no. 1, pp. 76-86, Feb. 2018

[14] K. Kampouropoulos, et al., "Multi-Carrier Optimal Power Flow of Energy Hubs by Means of ANFIS and SQP," IECON 2016 - 42nd Annual Conference of the IEEE Industrial Electronics Society, Florence, Italy, pp. 3990-3995, 2016.

[15] D. H. Tungadio, et al., "Power system state estimation solution using modified models of PSO algorithm: Comparative study," Measurement, vol. 92, pp. 508-523, 2016.

[16] M. Wang, et al., "GPU-based Power Flow Analysis with Continuous Newton's Method," 2017 IEEE Conference on Energy Internet and Energy Systems Integration, Beijing, China, pp. 1-5, 2017.

[17] A. Tazi, et al., "Firefly Algorithm for Solving Load Flow Problem," 2018 Renewable Energies, Power Systems and Green Inclusive Economy (REPS-GIE), Casablanca, Morocco, pp. 1-5, 2018.

[18] S. C. Kim and S. R. Salkuti, "Optimal power flow-based congestion management using enhanced genetic algorithms," International Journal of Electrical and Computer Engineering (IJECE), vol. 9, no. 2, pp. 875-883, Apr. 2019.

[19] M. N. M. Salleh, et al., "Adaptive Neuro-Fuzzy Inference System: Overview, Strengths, Limitations, and Solutions," International Conference on Data Mining and Big Data, pp. 527-535, 2017.

[20] M. Fikri, et al., "Power Flow Analysis by Numerical Techniques and Artificial Neural Networks," 2018 Renewable Energies, Power Systems and Green Indusive Economy (REPS-GIE), Casablanca, Morocco, pp. 1-5, 2018.

[21] M. Fikri, et al., "Power Flow Calculations by Deterministic Methods and Artificial Intelligence Method," International Journal of Advanced Engineering Research and Science (IJAERS), vol. 5, no. 6, pp. 148-152, 2018

[22] M. Fikri, et al., "Predicting Moroccan Real Network's Power Flow Employing the Artificial Neural Networks," 2019 Third International Conference on Intelligent Computing in Data Sciences (ICDS), Marrakech, Morocco, pp. 1-6, 2019.

[23] M. Fikri, et al., "Adaptive Neuro-Fuzzy Inference System to Predict the Voltage Magnitudes and Voltage Phase Angles," Proceedings of the 4th International Conference on ig Data and Internet of Things, pp. 1-7, 2019.

[24] L Powell "Power System Load Flow Analysis," The McGraw-Hill Companies, Inc., 2004.

[25] K. L. Priddy and P. E. Keller, "Artificial Neural Networks an Introduction," Tutorial Texts in Optical Engineering, SPIE Press, Bellingham, Washington, USA, vol. TT68, 2005.

[26] "Root Mean Square Error," [Online], Available: https://www.statisticshowto.datasciencecentral.com/rmse/. 